Information Jukebox - A semi-public

device for presenting multimedia information content.

published on netzspannung.org:

http://netzspannung.org/about/mars/projects/pdf/info-jukebox-2003-1-

en.pdf

21 March 2005

First published: Springer Journal "Personal and Ubiquitous Computing". Proceedings of the 1st International Conference on Appliance Design 2003. London: Springer Verlag, 2003, pp. 217-220. 


\title{
Information Jukebox - A semi-public device for presenting multimedia information content
}

\author{
Wolfgang Strauss, Monika Fleischmann, Jochen DenZinger, Christoph \\ GROENEGRESS AND YINLIN LI
}

MARS - Exploratory Media Lab

Fraunhofer Institute for Media Communicaton

Schloss Birlinghoven

53754 Sankt Augustin, Germany

http://imk.fraunhofer.de/mars

\{strauss, fleischmann, denzinger, groenegress, li\}@imk.fraunhofer.de

Fax: $\quad+49-[0] 2241-142133$

Phone: $+49-[0] 2241-143454$

\begin{abstract}
The Information Jukebox is a digital enquiry kiosk aimed at browsing media files. It combines a novel gesture-based and hands-free interface with an expressive graphical user interface and an attractive physical design. The system was developed within the context of a media arts exhibition and thus had to meet both functional and semiotic requirements. Unlike comparable systems it offers a complete and playful solution for browsing media.
\end{abstract}

Keywords: playful interface, information appliance, gesture-based interaction, disappearing computers, interface design, post-WIMP GUI.

\section{Introduction}

In this paper we introduce the Information Jukebox, a digital enquiry kiosk for exploring multimedia content. The Information Jukebox was developed as an installation within the context of a media art exhibition in 2002 and followed the purpose to represent previous works of the MARS - Exploratory Media Lab.

Unlike today's current black box-PCs and other information systems, the Information Jukebox is conceived and designed as an information appliance: the 
context defines the requirements and the Information Jukebox is specialized and limited to serve its purposes. Like the famous Wurlitzer jukebox, the Information Jukebox shows all of its content simultaneously. A novel gesture-based and touchless interface enables simple and natural access to the content.

\section{The MARS Information Jukebox: an installation for the media art exhibition 'Emoção art.ficial'}

In 2002, the cultural institution Itaú Cultural in São Paulo invited several media labs worldwide to present recent works in an exhibition. The MARS Exploratory Media Lab, directed by research artists Monika Fleischmann and Wolfgang Strauss, developed the Information Jukebox as a contribution for the exhibition "Emoção art.ficial” (Itaú Cultural 2002).

This context of an installation within a public exhibition on new media arts led to some basic requirements: On the one hand, of course, the overall aesthetic qualities had to be considered. In an environment such as this aspects of playfulness, joy and pleasure (Jordan 2002) are - in terms of the recipients' experience - as important as the traditional, current paradigm of usability. The recipient does not arrive with a concrete need (i.e. task) that ought be fulfilled in the best possible (i.e. most efficient) way, but with a certain interest and mixed expectations. Thus, the semantic qualities become as important as the mere technical functionalities. The Information Jukebox is self-referential, as it shows the work of a media lab within the work of a media lab.

Using a novel and playful post-WIMP interface to manipulate the Jukebox is an approach to meet the visitors' expectations. On the other hand, we had to satisfy 'traditional' requirements such as robustness and ease of use: Visitors of the exhibition encounter a great variety of installations and new interaction principles. Consequently, they spend a limited time exploring the installation. The exhibition was open for a period of two months, and technical support staff from our team was only present during the installation setup.

The Jukebox thus follows the information appliance approach, as it is easy to use and easy to maintain (Gellersen et al. 2001) and furthermore Norman's design principles of 'simplicity, versatility and pleasurability' (Norman 1999). It enlarges these with the moment of intuitiveness and natural interaction. 
In this sense the Jukebox was conceived and designed as a semi-public device that gives easy and fast access to existing media files showing previous work of the MARS-Lab (see Figure 1).

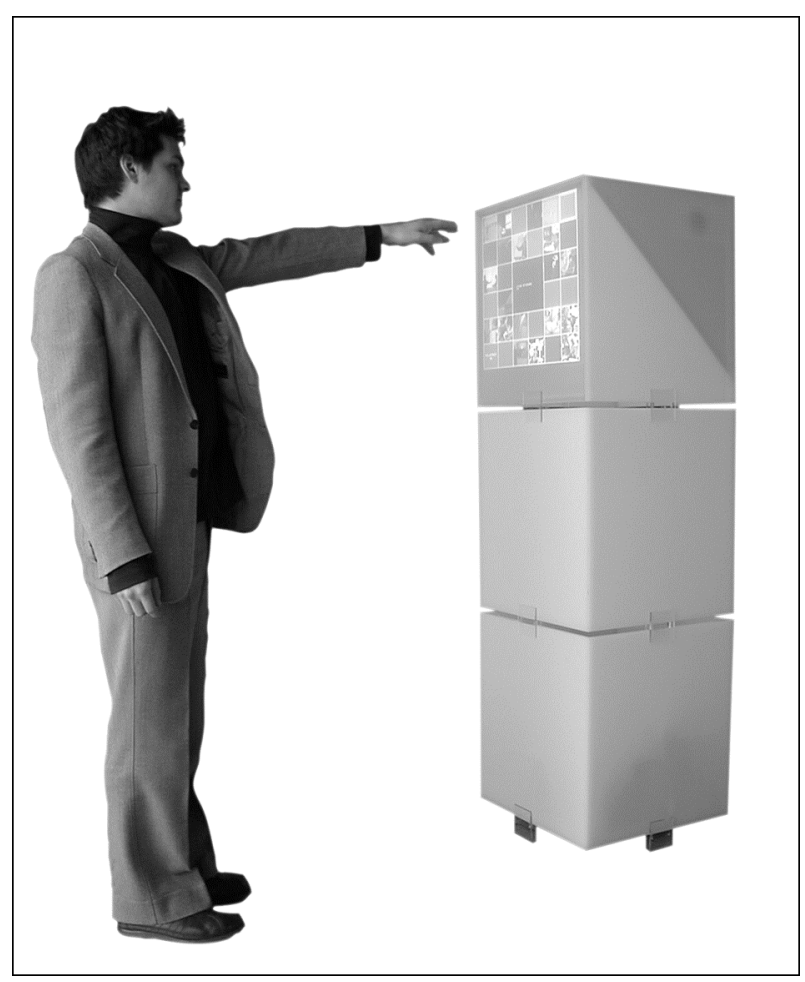

Figure 1: Information Jukebox

\section{User Interfaces}

As a result, the prerequisites for our interface are that it should be playful and pleasurable, easy to understand and to use, while being robust and easy to maintain. Also, due to the artistic inclination of the work, emphasis is placed on the appliance to make use of non-traditional user interfaces to provide information content and maximise information gain. So, it should provide a natural and intuitive way of interacting that neither restrains nor confuses the recipient, but rather enhances his experience.

\section{Electric Field Sensing}

It has been shown that interfaces based on electric field sensing (EFS) can be used for on-screen navigation without the need to touch or hold a device [Paradiso et al. 2000]. Reminiscent of the musical instrument developed by Lev Theremin in the first quarter of the $20^{\text {th }}$ century, EFS-based interfaces seem to overcome the necessity of touching or holding a device in order to manipulate digital objects (see Figure 2). The EFS-interface of the Information Jukebox was developed 
based on prior work and experiments with Theremins (Yelistratov et al. 1999; Fleischmann \& Strauss 2000; Geng et al. 2001). The 'Mars Bag' for example is a bag with an integrated Theremin. It actively indicates its own motion, as well as passviely the proximity of another person. In this case, motion is converted into sound and serves both as an expression in its own right and as a personal alarm system (Mars Bag 1999).

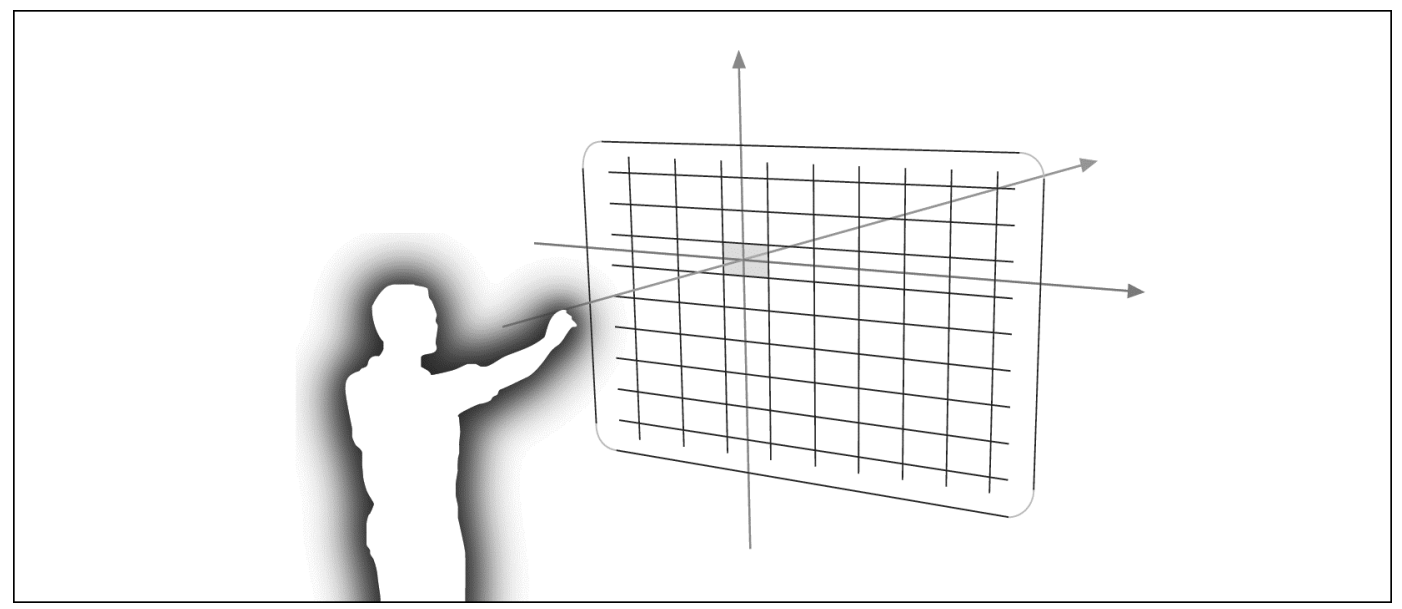

Figure 2: Touchless interface based on EFS.

Previous versions of EFS-based interfaces required the user to calibrate the system, which is no longer necessary in our advanced version installed in the Jukebox. Here, interaction takes place in a natural and invisible way by enhancing the energy field around the user's body. The interface is self-descriptive, it does not need instructions for use since moving one's body in front of the device results in visible feedback on the screen.

\section{Graphical User Interface}

Regarding the GUI, we aimed to design an attractive interface that is easy to use and provides a playful way of interaction with the system. Due to both, the limitations of EFS-based interfaces yielding slightly lower accuracy in determining the cursor position, and the exhibition context, the GUI itself was required to be very simple and lean. Since there was only a limited amount of media to be presented, we could lay out the GUI as a grid representing all of the content simultaneously. While keeping the design of the GUI in line with the overall physical design (discussed below), we arranged square-sized thumbnails so that they would form a larger square as shown in Figure 3. A box four times the size of the other thumbnails is placed in the middle of the screen for the purpose of presenting selected media files. In addition, we used bold colours to emphasize this layout as well as the playful installation character. 


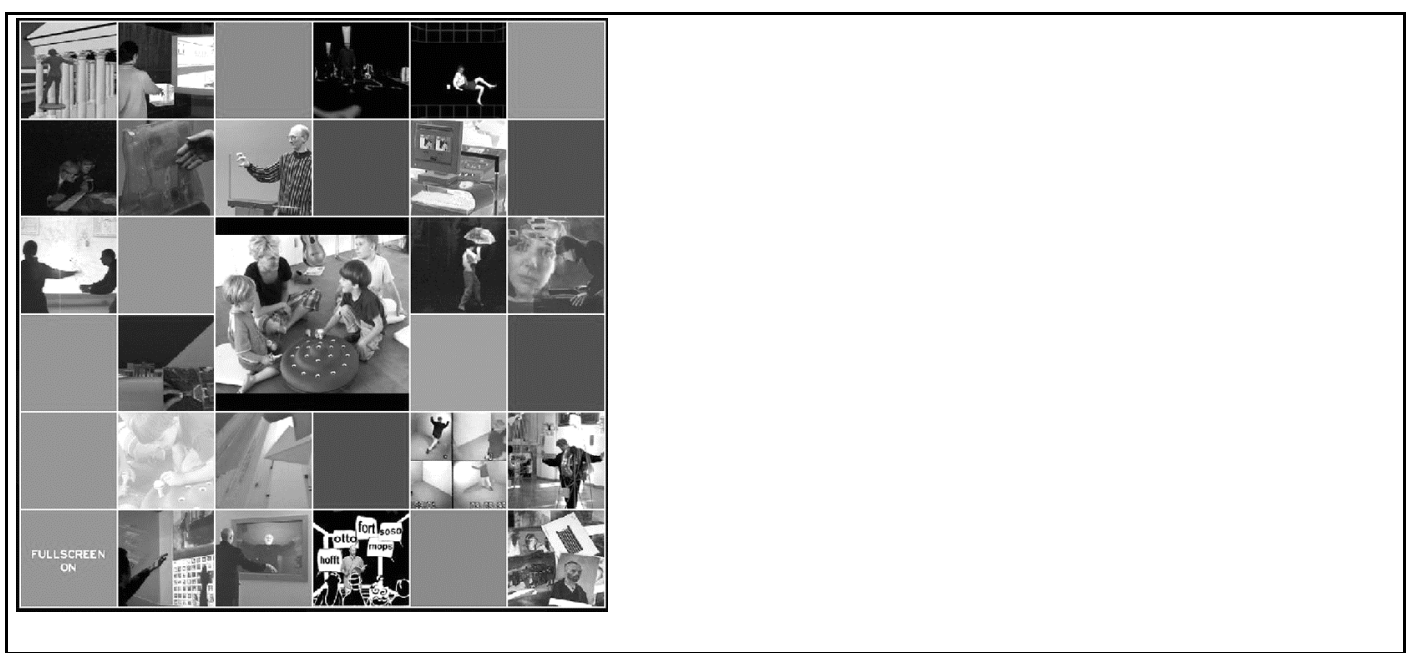

Figure 3: The Graphical User Interface

Essentially, the Information Jukebox supports two operations: cursor navigation and item selection. Information is represented by small images and users can select and view it in detail by pointing at the desired thumbnail. A square appears around the currently selected item to provide feedback for the user. Once an image is selected it is shaded in a bold colour indicating that is has been selected and the corresponding information is presented in the box at the centre.

As opposed to conventional file browsing systems, the GUI of the Jukebox has no depth, meaning that every piece of information that can be accessed via the interface is permanently visible and represented on the screen. This rule of flatness of the interface does indeed allude to the Wurlitzer Jukebox, which also shows all of its content (i.e. a list of songs and the singles themselves). The result of these considerations is that the complexity of the system is greatly reduced, though it also limits the functionality of the system and the maximum content it can provide. This latter point becomes less significant however, because of the relatively small amount of information we chose to present.

\section{Product Design}

As mentioned before, the aesthetics of the Information Jukebox had to be considered as an important aspect for the installation. Again, we had to meet functional as well as semiotic criteria.

The Jukebox consists of three cubes hovering above each other. A projector placed on the floor inside the base box points at the ceiling and the projection is reflected by a mirror held in the top box, towards one side of the upper box to 
produce a projection. The size of the Jukebox therefore arises from the distance necessary for the rear-projection inside the box.

On the one hand the division of the body into three cubes results in an impression of lightness, and on the other it has some advantage in terms of shipping and transportation

Regarding the material, we selected a white translucent Plexiglas, which connotes an idea of the immateriality of information. In addition, the cubes ironically refer to Mario Bellini's archetypical 1969 black box television set Brionvega Black ST 201.

The setup of the installation in São Paulo also implied a large projection besides the Jukebox itself. This public display mirrored the display of the jukebox and thus enabled a broader audience to view the installation (see Figure 4).

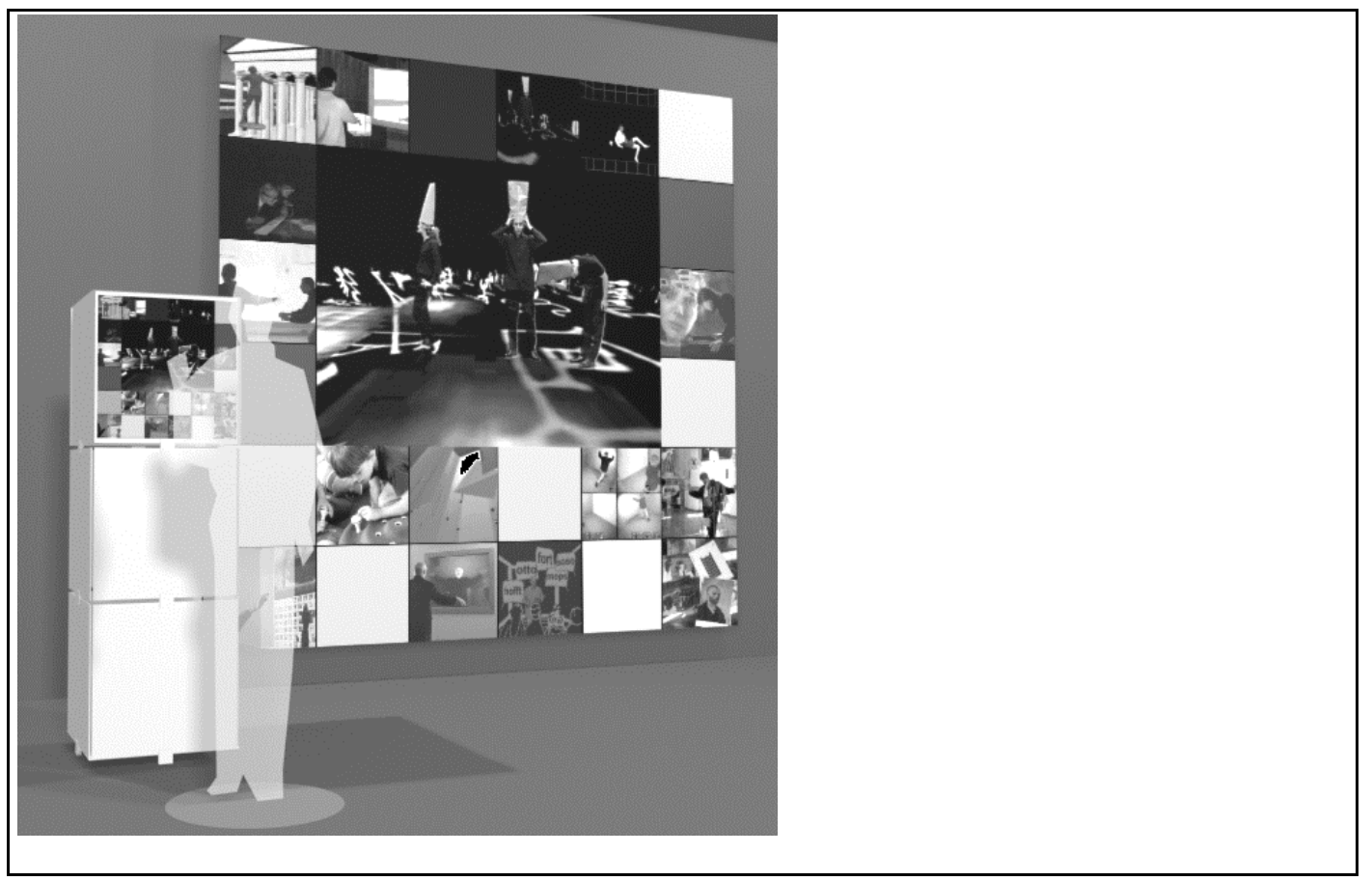

Figure 4: Possible setup with large public screen.

\section{Concluding Remarks}

Currently several areas of future research arise from the described work. Firstly, we plan to use the Information Jukebox in other application domains and therefore have to regard additional functionalities and possibly the use of additional interfaces. In each case, we will develop a custom graphical user interface according to the given context. 
Another, broader field to investigate will be the area of touchless interfaces and the further development of using body energy to manipulate computing devices. We already started first experiments by decomposing the Jukebox into a display area and an interaction area, which is a simple wireframe placed in space. The display area and the interaction area are physically separated.

Furthermore, we plan to combine the EFS-system with other interface and sensor technologies in order to ease interaction and experience.

Especially when considering the dawning era of ubiquitous or pervasive computing (Satyanarayanan 2002), we intend to deepen our research in this area. Particularly when picking up Weiser's rather socio-cultural than technical vision of a 'constant background presence' of the disappearing computer (Weiser 1991) and of 'calm technologies vanishing in the background' (Weiser \& Brown 1996), we have to develop novel, intuitive and unobtrusive interfaces.

\section{References}

Itaú Cultural (edt.) (2002), Emoção art.ficial, , Itaú Cultural, São Paulo, pp. 34-37.

Gellersen, H.-W., Reichsteiger, D., Schulz, K., Frick, O. \& Schmidt, A. (2001), 'Paper-to-Web: Papier als Eingabemedium für Formulare im World Wide Web', In: Oberquelle, H., Oppermann, R. \& Krause, J. (eds.), Berichte des German Chapter of the ACM: Mensch \& Computer 2001 - 1. Fachübergreifende Konferenz, Teubner, Stuttgart, Leipzig, Wiesbaden, pp 145-.

Geng, W., Yelistratov, V., Kolesnik, M., \& Strauss, W. (2001), 'Perceptual user interface for humancomputer interaction', In: Fleischmann, M. \& Strauss, W. (eds.), Proceedings Cast 01 // Living in Mixed Realities, Conference on Artistic, Cultural and Scientific Aspects of Experimental Media Spaces 2001, Fraunhofer IMK, Sankt Augustin,

Jordan, P.W. (2001), 'Pleasure with Products - The New Human Factors', In: Baumann, K. \& Thomas, B. (eds.), User Interface Design for Electronic Appliances, Taylor \& Francis, London and New York, pp.303341

Norman, D.A. (1999), The Invisible Computer, MIT Press, Cambridge MA \& London.

Paradiso, J.A., Hsiao, K., Strickon, J., Lifton, J.\& Adler, A. (2000). Sensor Systems for Interactive Surfaces. IBM Systems Journal Vol. 39 (3\&4).

Weiser M. (1991), "The Computer for the Twenty-First Century", in: Scientific American, September 1991, pp. 94-104 
Weiser M. \& Brown J.S. (1996), The Coming Age of Calm Technology,

http://www.ubiq.com/hypertext/weiser/acmfuture2endnote.htm (retr. 08-02-03)

Yelistratov, V., Fleischmann, M. \& Strauss, W. (1999). 'Two Approaches for Tangible Navigation in Virtual Environments', In : Proceedings of GraphiCon'99, 9th International Conference on Computer Graphics and Vision, Moscow, Russia, August 26 - September 1, 1999, reprinted: GMD Report No. 80, December 1999, http://www.gmd.de/publications/report/0080/ (retr. 08-02-03)

Fleischmann, M. \& Strauss, W. (2000), ‘Murmuring Fields oder ein Raum möbliert mit Daten’, in: Zacharias, W. (ed), Interaktiv - Medienökologie zwischen Sinnenreich und Cyberspace, München.

Satyanarayanan, M. (2002), "A Catalyst for Mobile and Ubiquitous Computing“, in: IEEE Pervasive Computing - Reaching for Weiser's Vision, Vol 1, Number 1, January-March 2002, pp. 2-5

Mars Bag (1999) -- http://www.nada.kth.se/erena/marsbag.html (retr. 08-02-03) 